\title{
Epidemiology of yaws: an update
}

This article was published in the following Dove Press journal:

Clinical Epidemiology

2 April 2014

Number of times this article has been viewed

\section{Walter M Kazadi' \\ Kingsley B Asiedu² \\ Nsiire Agana ${ }^{3}$ \\ Oriol Mitjà ${ }^{4,5}$}

'Office of the WHO Representative for Papua New Guinea, World Health Organization, Port Moresby, Papua New Guinea; ${ }^{2}$ Department of Control of Neglected Tropical Diseases, World Health Organization, Geneva, Switzerland; ${ }^{3}$ Public Health Division, Ghana Health Service, Accra, Ghana; ${ }^{4}$ Barcelona Centre for International Health Research, Hospital Clinic, University of Barcelona, Barcelona, Spain; ${ }^{5}$ Lihir Medical CentreInternational SOS, Newcrest Mining, Lihir Island, Papua New Guinea
Correspondence: Oriol Mitjà

Department of Community Health, Lihir Medical Center Post Office Box 34, Lihir Island, NIP, Papua New Guinea

Tel +34619741277

Fax +6759864288

Email oriolmitja@hotmail.com
Abstract: Yaws, a neglected tropical disease, is targeted for eradication by 2020 through large-scale mass-treatment programs of endemic communities. A key determinant for the success of the eradication campaign is good understanding of the disease epidemiology. We did a review of historical trends and new information from endemic countries, with the aim of assessing the state of knowledge on yaws disease burden. Transmission of yaws is now present in Africa, Asia, and the South Pacific. At least 12 countries are known to harbor yaws cases and 21 to 42 million people live in endemic areas. Between 2008 and 2012 more than 300,000 new cases were reported to the World Health Organization. Yaws presented high geographical variation within a country or region, high seasonality for incidence of active disease, and evidence that low standards of hygiene predispose to suffering of the disease. Key data issues include low levels of reporting, potential misdiagnosis, and scarce documentation on prevalence of asymptomatic infections. Currently available data most likely underestimates the magnitude of the disease burden. More effort is needed in order to refine accuracy of data currently being reported. A better characterization of the epidemiology of yaws globally is likely to positively impact on planning and implementation of yaws eradication.

Keywords: eradication, Treponema pertenue, endemic countries, prevalence, incidence

\section{Introduction}

Yaws is a neglected tropical disease caused by Treponema pallidum subspecies pertenue. This bacterium causes a chronic relapsing nonvenereal treponematosis characterized by highly contagious primary and secondary cutaneous lesions and noncontagious tertiary destructive lesions of the bones. The infection may become latent at any time and remain asymptomatic for several years with only serologic evidence of infection. In about $10 \%$ of cases, late reactivation leads to bone lesions, deformities, and disability. Yaws is usually diagnosed clinically, but the diagnosis is difficult and requires serological confirmation. ${ }^{1}$

The World Health Organization (WHO) aims to eradicate yaws by 2020 through adoption of the Morges strategy, which consists of initial mass treatment of endemic communities followed by surveys every 6 months to actively detect and treat remaining cases and their contacts. ${ }^{2}$ The use of a single oral dose of azithromycin ${ }^{3}$ has made mass treatment more feasible and is currently recommended in areas where yaws is prevalent. One of the key determinants for the success of the eradication campaign is good understanding of the disease epidemiology, particularly its geographic distribution and risk factors. 
The aim of this review is to provide a better characterization of yaws epidemiology worldwide based on an analysis of historical trends and some of the new information from endemic countries. Key issues related to yaws epidemiology, and gaps in knowledge about location of cases and their implications for yaws eradication, are also discussed.

\section{Risk factors for yaws}

Yaws has been known to affect populations living between the Tropic of Cancer and the Tropic of Capricorn where warm, humid, and moist climates provide favorable environments for the infection to spread. Yaws flourishes best in countries with the mean annual isotherm of $27^{\circ} \mathrm{C}$ and over. The disease does not thrive in colder climates or in climates where there are extremes of temperature. Despite ample opportunities for it to be carried from the tropics to more temperate climates, this infection has not given rise to infectivity. ${ }^{4}$

The highest incidence of yaws within the tropics appears to be intimately bound to a heavy rainfall. In general, the high incidence of yaws occurs in areas where the average rainfall is above $1,300 \mathrm{~mm}$ per year. Also, yaws has a clear seasonality and the number of cases tends to increase during the rainy seasons. ${ }^{4}$ A high rainfall usually entails higher occurrence of typical yaws cases; this is made up of an increase in new cases or reinfections, and of a higher relapse-rate of old cases. Of note, Saunders et $\mathrm{al}^{5}$ reported striking differences in the lesions during the wet and dry seasons. In the rainy season a greater proportion of cases showed open infectious lesions and papillomatous frambesides. During the dry season, they noticed that the eruptions are frequently drier, scalier, and of maculopapular type.

Yaws cases appear to affect mostly to children between 2 and 15 years old, who are also considered as the reservoir for infections. ${ }^{6}$ It appears that rather more males than females suffer from the disease, which is most usually acquired after a cut or abrasion in the lower legs. The explanation is that boys are more active than girls and therefore suffer more traumas. It is considered that transmission occurs through direct skin contact with a fluid from an infected lesion. Children born to mothers affected with yaws are generally unaffected and most of the evidence seems to indicate that the disease is not acquired congenitally.

The disease is one that primarily attacks rural communities, particularly those in poor economic circumstances and with low standards of hygiene, with incidence declining as social and economic status rise. In addition, in such rural settings, there usually are extensive areas of vegetation (bush), which increases the chances of injury to legs and feet and, therefore, liability of infection.

\section{Historical data}

In 1948, when WHO was established, endemic treponematoses were among the major public health problems that the new agency had to deal with. A review of the historical and current literature ${ }^{7}$ from 1950 to 2013 indicates that at least 90 countries have reported yaws (Table 1; Figure 1). ${ }^{8}$ It was estimated that there were, globally, between 50 and 150 million cases of active yaws in the early 1950s. The countries in which the disease was prevalent in South America included Venezuela, Bolivia, Colombia, Ecuador, and Brazil. The heaviest burden of yaws in this part of the world was in Haiti and other Caribbean Islands. Passing to Africa, the disease was highly prevalent in most countries of the west coast as well as in Uganda, Mozambique, and Madagascar. ${ }^{9}$ In the 1950s, about 20 African countries reported more than 10,000 cases per annum each, and four countries more than 100,000 cases each including Côte d'Ivoire, Gold Coast (Ghana), Cameroon, and the Belgian Congo. In Asia, it was very common in Thailand and Indochina (ie, Cambodia, Laos and Malaysia), and it was also found in some districts of India and China. Finally, yaws was also present in the South Pacific, including the north of Australia, and was highest in incidence in the smaller islands, being a problem in Papua New Guinea and the Solomon Islands.

The World Health Assembly adopted resolution WHA2.36 $6^{10}$ in 1949 to support control of this disease. The initial WHO-assisted pilot projects ${ }^{11}$ to introduce penicillin in mass treatment campaigns in Bosnia, Haiti, Indonesia, the Philippines, and Thailand were rapid and remarkably successful. The spectacular results achieved with singledose treatment helped to reinforce community cooperation in the campaigns. Between 1952 and 1964, WHO and the United Nations Children's Fund supported mass treatment

Table I Status of yaws endemic countries according to WHO, 2013

\begin{tabular}{llll}
\hline WHO region & $\begin{array}{l}\text { Endemic } \\
\text { (status known } \\
\text { 20I3)* }\end{array}$ & $\begin{array}{l}\text { Previously } \\
\text { endemic (status } \\
\text { unknown 20I3) }\end{array}$ & Total \\
\hline African & 8 & 32 & 40 \\
Americas & 1 & 27 & 28 \\
Southeast Asia & 2 & 9 & $1 \mathrm{I}$ \\
Western Pacific & 3 & 8 & $1 \mathrm{I}$ \\
Total & 14 & 76 & 90 \\
\hline
\end{tabular}

Notes: *12 endemic countries: Benin, Cameroon, Central African Republic, Republic of the Congo, Côte d'Ivoire, Democratic Republic of the Congo, Ghana, Togo, Indonesia, Papua New Guinea, Solomon Islands, and Vanuatu; two countries certified elimination: India, Ecuador. Adapted with permission from Global Health Observatory Data Repository: Yaws [webpage on the Internet]. Geneva: World Health Organization; 2013. Available from: http://apps.who.int/gho/data/node.main. NTDYAWS?lang $=$ en. $^{8}$ 


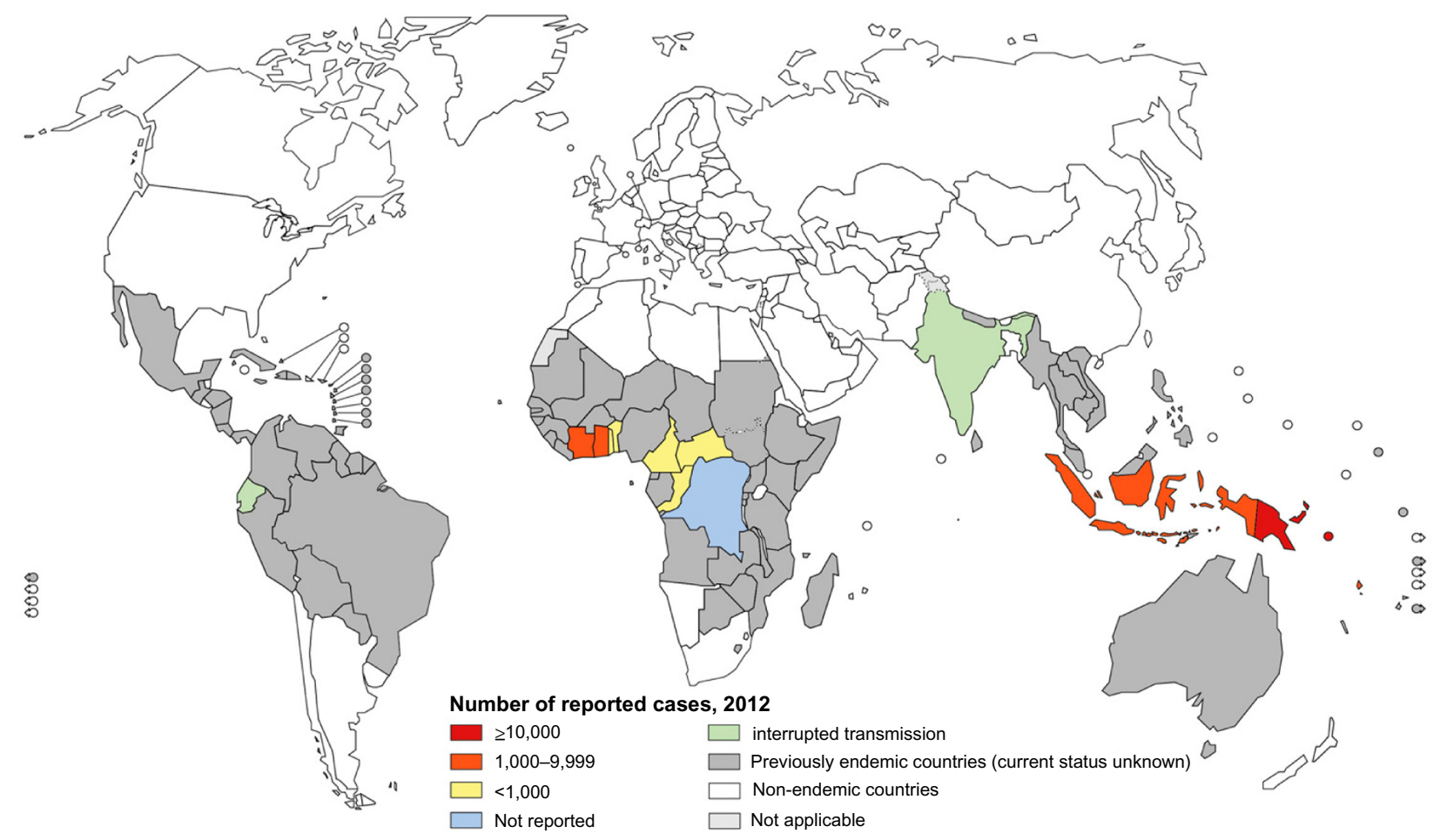

Figure I Distribution of yaws worldwide 2012.

Notes: Figure shows the most recent data from routine surveillance in yaws endemic countries (colour-shaded) compared with the global distribution in 1950 (gray-shaded). Reproduced from http://www.who.int/yaws/epidemiology/en/ with permission of the World Health Organization.

campaigns using injectable penicillin in 46 of the 90 countries known to be endemic. About 300 million people were screened and over 50 million cases and contacts were treated. By the end of the campaign, the global burden of cases of endemic treponematoses was estimated to be reduced by $95 \%$ to a mere 2.5 million cases. ${ }^{6}$

Figure 1 illustrates how the yaws map shrank after the 1950s campaign (color-shaded countries) compared to the precampaign period (gray-shaded countries). Whether this is a true reflection of the actual yaws epidemiology or burden may be questionable. However, the endemic areas of the 1950s may provide the basis for previously endemic countries to determine yaws status for the purpose of planning the new eradication efforts. Countries may be classified into one of three possible categories: 1) yaws is still present; 2) yaws is absent; or 3 ) the status of yaws is uncertain.

Little activity was undertaken from the late 1960s to the early 1990s, and formal reporting of yaws from a number of countries to WHO stopped. The Organization also did not have any formal system to verify interruption of transmission and certify countries. Only 14 countries kept yaws activities on their public health agendas and, of these countries, two (Ecuador ${ }^{12}$ and India ${ }^{13}$ ) reported interrupting transmission of the disease in 2003; albeit formal verification by WHO is still pending. The other 12 known endemic countries (Benin, Cameroon, Central African Republic, Republic of the Congo, Côte d'Ivoire, Democratic Republic of the Congo, Ghana, Togo, Indonesia, Papua New Guinea, Solomon Islands, and Vanuatu) need technical assistance and resources to eradicate the disease. The current status of the remaining 76 countries that were endemic in the 1950s should also be reestablished.

\section{Remapping the disease}

Between 21 and 42 million people are estimated to live in tropical areas where yaws is endemic. There is robust evidence that at least three WHO regions have yaws transmission and that there are at least 12 yaws endemic countries (Table 1). Global estimates are unclear, but, from 2008 to 2012 more than 300,000 new cases were reported to WHO. ${ }^{2}$ Due to a series of factors, including poor disease surveillance, low level of reporting, difficulties in diagnosis, and inconsistence in the comparative analyses, the true incidence and impact of yaws is uncertain and likely significantly higher than currently reported.

Importantly, scarce published data are available; during the past 20 years only about 26 papers have been published on yaws epidemiology ${ }^{14-40}$ and some of these papers deal with small numbers of cases. Published data on scientific 
journals are insufficient to provide information on the extent and nature of the yaws problem worldwide. WHO convened a consultation on yaws in March 2012 and the summary report of the meeting serves as additional information for this epidemiological update. ${ }^{2}$

Below, we summarize data of published studies on systematic surveillance of populations with yaws. We extracted data on prevalence of yaws lesions based on dermatological examination with or without serological confirmation and estimated 95\% confidence intervals (CIs) for prevalence rates. We also report data on incidence based on the number of yaws cases reported by country to WHO and published in the Global Observatory Data Repository. We present data by WHO region.

\section{Yaws in the WHO African region}

Although the full extent of yaws is not known in the African region, seven countries reported cases of yaws to WHO in 2012 - Benin, Cameroon, Central African Republic, Congo, Côte d'Ivoire, Ghana, and Togo - and one in 2008: Democratic Republic of the Congo (Table 2). Ghana appears to be the most endemic country, reporting more than 20,000 cases annually from 2007 to 2010 (Figure 2). Another hot spot of yaws in Africa is the tropical forest inhabited by the indigenous populations (Pygmies) along the Congo, Cameroon, and Central African Republic borders. At least, three countries have ongoing national programs to fight yaws - Ghana,
Cameroon, and Congo - and report yaws using the national health information system (Figure 2).

Surveys carried out in Ghana in 2008 showed a national clinical prevalence of yaws lesions at $0.68 \%$; however, prevalence in some rural communities was as high as $20 \%{ }^{2}$ The general trend of case notification in Ghana showed a substantial decline in the number of cases over time (from 35,200 in 2009 to 9,300 in 2012; Figure 2). ${ }^{8}$ All ten regions of Ghana report yaws annually, but the Eastern, Central, Western, Volta, and Ashanti regions are the most affected places. From the routine reporting, only 19 out of the 170 districts have not reported yaws from 2008 to 2011. The metropolitan cities of Accra and Kumasi are not seeing yaws and most districts in the Northern Region have not been submitting reports.

In Cameroon, the result of the 1950s mass-treatment campaigns had reduced to an extent that everyone thought there was no longer yaws in the country. Unfortunately, in 2007 and 2008, there were outbreaks of yaws among the indigenous populations in the Lomié health district. In general, the indigenous populations live in poor areas with little or no access to health care and schooling. They are migrant populations who live in the forest. This prompted the Ministry of Health to include yaws into the leprosy, leishmaniasis, and Buruli ulcer control program in 2009. Further activities carried out in 2010 identified 789 suspected cases of yaws. Today, all health districts are considered to be endemic; however, the great majority of the cases are reported from the East

Table 2 Countries with available information on yaws, 2008-20I2

\begin{tabular}{|c|c|c|c|c|c|}
\hline \multirow[t]{2}{*}{ Country } & \multicolumn{2}{|c|}{ Number of cases ${ }^{a}$} & \multicolumn{3}{|c|}{ Prevalence of yaws lesions in cross-sectional surveys } \\
\hline & Year of report & Number & Year of survey & Sample size/district & $\begin{array}{l}\text { Prevalence, \% } \\
(95 \% \mathrm{Cl})\end{array}$ \\
\hline \multicolumn{6}{|l|}{ Africa } \\
\hline Benin & 2012 & II & 2013 & 900 (Toffo, Ze, Allada) $^{c}$ & I.I (0.8-I.3) \\
\hline Cameroon & 2012 & 16 & 2012 & I,075 (Lomie) & $9(7.5-10.9)$ \\
\hline Central African Republic & 2012 & 230 & 2012 & 2,030 (Lobaye) $^{d}$ & $11.3(10.2-12.8)$ \\
\hline Republic of the Congo & 2012 & 197 & 2013 & 6,215 (Bétou, Enyelle) & $2.9(2.6-3.4)$ \\
\hline Côte d'lvoire & 2012 & 2,864 & 2007 & 2,182 & $0.5(0.28-0.90)$ \\
\hline Democratic Republic of the Congo & 2008 & 383 & 2012 & I, I 76 (Wasolo) & $4.7(3.7-6.1)$ \\
\hline Ghana & 2012 & 9,356 & 2008 & ND & 0.68 (NA) \\
\hline Togo & 2012 & 5 & 1994 & ND & $0.23(\mathrm{NA})$ \\
\hline \multicolumn{6}{|l|}{ South East Asia } \\
\hline Indonesia & 2012 & 3,476 & 1991 & 37,000 (Sumatra) & $0.31(0.26-0.37)$ \\
\hline Timor Leste ${ }^{b}$ & NA & ND & 2010 & I,535 (Oe-cusse) & $0.39(0.18-0.85)$ \\
\hline \multicolumn{6}{|l|}{ Pacific } \\
\hline Papua New Guinea & 2012 & 17,560 & 2001 & 227 (Port Moresby) & $14.5(10.5-19.7)$ \\
\hline Solomon Islands & 2012 & 12,372 & 2007 & ND & 0.4 (NA) \\
\hline Vanuatu & 2012 & 1,718 & 1989 & 20,200 (Tanna) & $2.3(2.1-2.5)$ \\
\hline
\end{tabular}

Notes: a Number of cases of yaws more recently reported by country; adapted with permission from http://www.who.int/yaws/epidemiology/en/; bcountry where yaws is known to be endemic but with no incidence data available between 2008 and 2012; 'Ayelo G, personal communication, 2013; 'Boua B, personal communication, 20I3. Data from WHO, ${ }^{2}$ Global Health Observatory Data Repository: Yaws, ${ }^{8}$ Coldiron et al, ${ }^{14}$ Manirakiza et al, ${ }^{15}$ Edorh et al, ${ }^{21}$ Akogun et al, ${ }^{23}$ Backhouse et al, ${ }^{28}$ Mitjà et al, ${ }^{13}$ de Noray et al. ${ }^{36}$ Abbreviations: $\mathrm{Cl}$, confidence interval; NA, not applicable; ND, no data. 

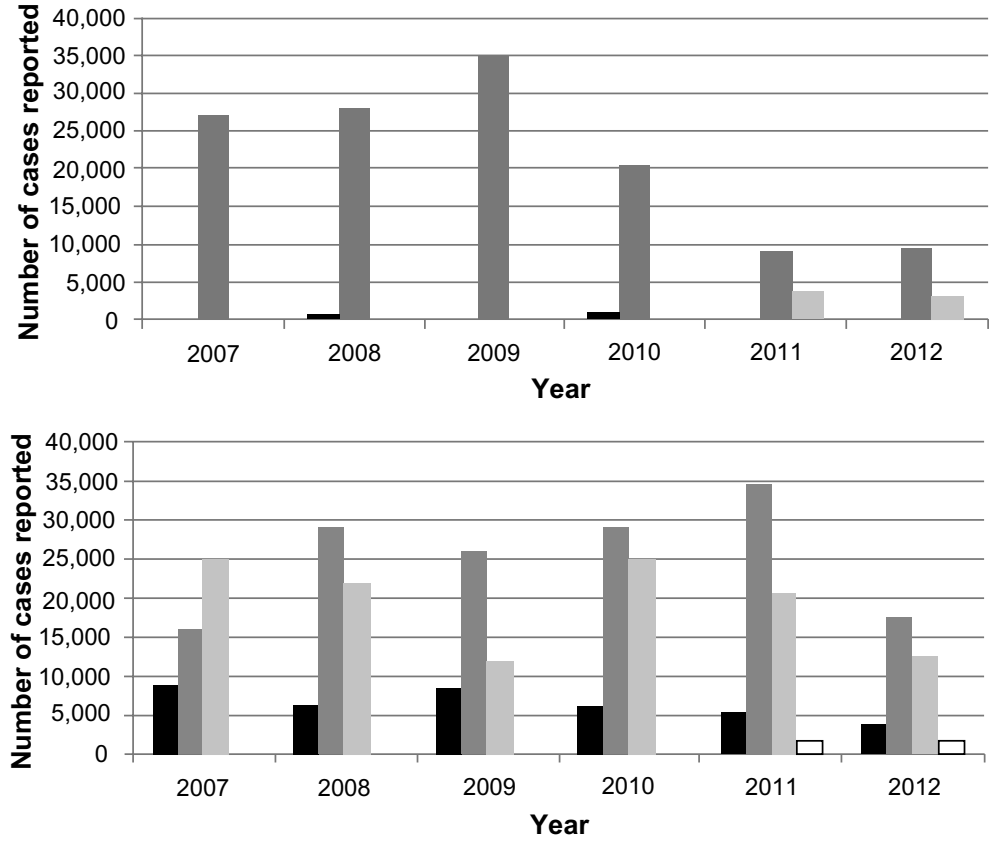

Figure 2 Trends in number of yaws cases in three African countries and four countries in South-East Asia/Western Pacific. Note: Data from World Health Organization. ${ }^{8}$

Region. In 2012, a survey to determine the current prevalence of yaws lesions in the Lomié health district showed that yaws remains highly endemic among Baka indigenous populations as well as Bantu communities, with a prevalence rate of yaws lesions of $9 \%$ (95\% CI: $7.5-10.9){ }^{2}$

In the Republic of the Congo, there are three endemic regions - Lékoumou, Likouala, and Sangha - but at least 16 of 84 districts nationwide report yaws cases annually. The most affected group of the population is the indigenous people (Babengas) who live in the humid forested region of Likouala. These regions are contiguous with the endemic foci in Cameroon where the indigenous population live. Coldiron et al conducted a screening and treatment program in two districts of Likouala region (Bétou and Enyellé) in 2013. ${ }^{14}$ The program reached some 6,000 children and identified 485 clinical cases with yaws-like ulcers. 183 subjects had confirmed reactive serology, representing a $2.9 \%$ (95\% CI: $2.6-3.4)$ prevalence of clinical yaws.

The yaws control program in Central African Republic was reestablished in 2012. Recent evidence of surveys indicate that the underlying prevalence of $T$. pallidum infection in the communities of southwest Central African Republic (Lobaye and Sangha) may be very high with $11 \%$ (95\% CI: 10.2-12.8) of schoolchildren with yaws skin lesions and $85 \%$ (95\% CI: $82.5-88.1)$ prevalence of reactive serology (active and latent) (B Boua, personal communication, March 2013). This region, close to the borders of the Democratic
Republic of the Congo and the Republic of the Congo, is also inhabited by indigenous populations. ${ }^{15}$ Previous serological surveys performed in 1978 in the same areas showed that $78 \%-86 \%$ of children had sera positive for Treponema, suggesting massive endemicity of T. pallidum pertenue infection. ${ }^{16,17}$ Another serological survey in 1992 reported 20\% reactive serology prevalence rates in Lovaye. ${ }^{18}$ In 1993, Louis et al reported that indigenous populations in Central African Republic were still infected with yaws, and they emphasized the risk for the spreading of the disease among settled communities because of the trend of these people to abandon their nomadic life style. ${ }^{19}$

Presently, efforts are being made to assess the level of endemicity of clinical and serological yaws in the communities and to map the populations in some other African countries. In 2012, Côte d'Ivoire notified 2,864 cases originating from $60 \%$ of the health districts; Touré et al had reported that active yaws prevalence in Côte d'Ivoire was 0.5\% (95\% CI: 0.28-0.90) in 2007 based on available hospital statistics and a cross section investigation. ${ }^{20}$ In Benin, a cross sectional study carried out in 2012 in schoolchildren of the Toffo, Allada, and Ze health districts found prevalences of yaws skin lesions of $1.1 \%$ (95\% CI: $0.8-1.3)$ and reactive serology of 2.4\% (95\% CI: 1.6-3.7) (G Ayelo, personal communication, March 2013). In Togo, only 15 yaws cases were notified in the south of the country in 2010; though, in 1994, Edorh et al reported about 5,000 annual yaws cases among children. ${ }^{21}$ In Democratic Republic of the Congo, 
a community-based survey identified an overall prevalence of yaws skin lesions of $4.7 \%$ (95\% CI: 3.7-6.1) in the Wasolo health zone, Equator province in 2012. ${ }^{22}$ In Sierra Leone, 93 cases were reported in 2003 in remote rural communities of Bombali and Port Loko districts in the northern region, though there has been no survey done on yaws and no updated surveillance data is available at this stage.

A yaws outbreak was reported in northeastern Nigeria in 1998 by Akogun, ${ }^{23}$ despite the official position that yaws had been eradicated in Nigeria. A more recent study by Nnoruka, however, failed to identify any yaws-affected individuals among 2,871 consecutive patients at the dermatology clinic of the University of Nigeria in Enugu (approximately 500 miles from the more rural area of Garkida, where Akogun ${ }^{23}$ conducted his research). ${ }^{24}$ In this study, however, most of the patients were adults, diagnosis was based on the patient's history and physical examination, and no tests to specifically identify treponemes or treponemal infection were performed. ${ }^{24}$

\section{Yaws in the WHO Southeast Asia region}

In the Southeast Asia region, Indonesia reported 3,476 cases in $2012,{ }^{2}$ and no official data are available from Timor-Leste, although this is known to be a country where yaws remains endemic (Table 2). Little information on yaws after the 1960s is available from other countries in the region such as Cambodia, Laos, Malaysia and the Philippines that were previously endemic. A significant reduction of prevalence to near elimination was found in these countries during resurveys in the 1960s and later in the 1980s, suggesting that yaws' disappearance could be expected. The estimated incidence in Timor Leste ranges from 500 to 1,000 cases per year and the disease has been reported from at least 6 of 13 districts in recent years. ${ }^{41}$ In 2010 a cross sectional survey in four districts in Timor-Leste showed a prevalence of yaws lesions in children and adults of $0.39 \%$ (95\% CI: $0.18-0.85)$. In Indonesia, the incident number of cases detected from 2001-2011 has varied significantly over time (Figure 2). The general trend showed an increase up to 8,907 cases in 2007, when the number of cases reached the highest level reported. Thereafter, in 2008, the number of cases diminished significantly to 6,083 , and has continued to decrease in 2010, 2011, and 2012 with 6,128, 5,319, and 3,746 cases per annum, respectively (Figure 2 ). Sixty-eight (14\%) of the 497 districts of Indonesia report yaws; most cases are found in the eastern part of Indonesia. A hot-spot for yaws is the province of Nusa Tenggara Timur, consisting of 566 islands, where 2,800 cases were reported in 2012
(C Widaningrum, personal communication, March 2013). Other endemic provinces are South East Sulewasi, Papua and Maluku. There are no recent seroprevalence published studies about yaws in Indonesia, except for a study carried out in West Sumatra in 1991 that concluded that prevalence of yaws lesions was $0.3 \%$ (95\% CI: $0.26-0.37) .{ }^{28}$ Indonesia has targeted elimination of yaws by 2017 (following global target) using active and passive case detection and prompt treatment; some of the major problems faced by the program include the difficulty of reaching endemic communities scattered over many small islands.

Great success was achieved in India, where no cases of yaws have been reported since 2003 and elimination was officially declared on September 19, 2006. ${ }^{13}$ This success is the result of an active government-backed elimination program launched in 1996 and based on active case finding with treatment of infectious and latent cases and contacts with benzathine penicillin. In India, toward the final stage of the campaign, a cash reward system was established to encourage voluntary reporting of any suspected case. The successful Indian elimination campaign showed the world that yaws prevalence in a defined area can be brought to zero through deliberate efforts and demonstrated that yaws eradication (ie, the reduction to zero of yaws prevalence on a global scale) is an attainable goal if sufficient resources and political commitment are available.

\section{Yaws in the WHOWestern Pacific region}

Prior to the 1950s campaigns, there was a high burden of yaws in the Western Pacific region. ${ }^{42}$ Following mass treatment, however, the number of reported cases declined significantly to the point that yaws was considered eliminated in most areas. ${ }^{29}$ Since the late 1970 s, however, reports of suspected cases of yaws from several areas of Papua New Guinea, ${ }^{30,31}$ the Solomon Islands, ${ }^{32,33}$ and Vanuatu ${ }^{34-36}$ have appeared frequently in the literature, suggesting that elimination was not achieved. Yaws is not a notifiable disease in the region but, in 2012, Papua New Guinea reported 17,560 yaws cases, followed by the Solomon Islands, 12,372 cases, and Vanuatu, 1,718 cases (Table 2).

In Papua New Guinea, prevalence of yaws lesions in the most recently carried out studies range between $4 \%$ (95\% CI: 2.8-6.0) in Karkar islands in $1990^{28}$ and 15\% (95\% CI: 10.5-19.7) in the peri-urban settlements of Port Moresby in $2001 .{ }^{30}$ Hospital-based incidence estimates are among the highest rates in the world (2.5\% per year). Systematic national records on yaws have been available since 2000, showing that transmission is active and currently occurring in most parts 
of Papua New Guinea. There are accounts of yaws in all the coastal areas and in some inland lowland areas (ie Simbu Province). The government has reported over 20,000 cases per year during the last 5 years. The most affected provinces are the New Ireland Province, East and West New Britain (which reported more than 5,000 cases each in 2011), and, to a lesser extent, Northern province, Madang, and West Sepik. The Papua New Guinean government is now interested in reactivating intensified yaws control activities (W Houinei, personal communication, March 2013).

In the Solomon Islands, between 1998 and 2012, all ten provinces reported cases of yaws detected among patients attending primary health care facilities. The absolute number of cases in 2012 was estimated to exceed 12,000; more than 4,000 being diagnosed in the Western Province. The incidence rates have fluctuated in the past 10 years, but have shown a downward trend from a peak rate of 65 cases per 1,000 per year, reaching its lowest point in 2012 (23 cases per 1,000 per year). ${ }^{2}$ The incidence rate is high in Makira and Guadalcanal, and low in Isabel and Choiseul.

In Vanuatu, 2,197 cases of yaws were reported in 2011 and 2,154 in 2012. ${ }^{8}$ The highest endemicity is found in the Provinces of Tafea and Sanma (F Taleo, oral communication, March 2013). Despite repeated mass treatments carried out in these provinces, clinical cases continue to be reported. Cases are commonly found in clusters on certain islands of Tafea (ie,Tanna Island) and Sanma (ie, Santo Island) provinces. A serological and clinical survey using a cluster sampling method was conducted in 2008 to assess the endemicity of yaws on Tanna island. Among 306 individuals from whom blood samples were taken, 95 (31\%, 95\% CI: 26.1-36.4) were positive for rapid plasma reagin and/or rapid diagnostic test for syphilis. ${ }^{34}$ These results were similar to those reported by de Noray et al in 2001 in the Santo Island, were a prevalence of reactive serology of 20.9 (95\% CI: 16.5-26.1) was found. ${ }^{36}$ Data from other areas of the South Pacific are very limited. Surveys conducted in the 1950 s-60s showed a varying degree of yaws endemicity in the region with a high prevalence found in Fiji. However, passive surveillance continued in these areas, and a very small number of cases were reported over the next few decades. A recent survey in Wallis and Futuna (Polynesia) found only one adult with skin lesions consistent with yaws. The authors suggest that yaws has not resurged in these islands. ${ }^{43}$

In Australia, the most recent information is from 1972 , reporting a serological survey in the aboriginal population of the Northern Territory. The authors concluded that while no case of active yaws was found, the prevalence of syphilis reactive serology varied from $3.4 \%$ to $58 \%$ indicating that yaws and probably venereal syphilis were present. ${ }^{44}$

\section{Yaws in the WHO region of the Americas}

There is scarce recent information on the epidemiological situation of yaws in the WHO region of the Americas where, since 1977, this disease has no longer been identified as a public health problem. ${ }^{45}$ Yaws incidence had been very low since 1970 with small foci remaining in Ecuador, Suriname, Guyana, Colombia, and some islands of the Caribbean. ${ }^{45}$ Importantly, it is not known whether a lack of reported cases is the result of true disease elimination or lack of surveillance.

In Ecuador, a survey in the northern region of Esmeraldas documented a prevalence of active yaws of $16.5 \%$ (95\% CI: 14.9-18.3) in $1988 .{ }^{42}$ A continuous long-term communitybased surveillance program was therefore put in place focusing on yaws as one of the sentinel diseases. The successful results of this intervention were consistently reported in a second survey in 1993, showing a reduction in the prevalence of clinical cases to $1.4 \%$ (95\% CI: $0.88-2.3) .{ }^{40}$ In Ecuador no yaws cases were identified between 1993 and 1998; therefore, the disease is also regarded as being eliminated. ${ }^{12}$ In Guyana, following the implementation of a control program in 2000, a resurvey conducted the following year showed a drop in prevalence of yaws skin lesions from precampaign 5.1\% (95\% CI: $3.9-6.6)$ to postcampaign $1.6 \%$ (95\% CI: $0.08-0.3) .{ }^{38}$

\section{Characterizing epidemiology of yaws: key issues, challenges, and implications for yaws eradication program Low completeness and timeliness of reporting}

One of the key challenges facing yaws eradication today is the lack of clear understanding of the disease burden and distribution. Several conditions contribute to this situation, namely: 1) yaws is not a notifiable disease, so countries do not need to report to WHO; 2) the assessment of incidence at a national level relies on reports being submitted to the National Health Information System - therefore, underreporting may have a major impact on incidence estimation; and 3) figures of incidences are largely based upon returns of attendances at clinics and rural dispensaries. In many such establishments, the standards of diagnosis are low and do not have serologic methods to confirm a clinical suspicion. Often, diagnosis is based upon clinical observation only, so misdiagnosis may undermine the actual estimate of disease 
burden. Also, this would not account for the many patients who are in the latent stage. All in all, current figures on yaws incidence can be used only as a rough indication.

We have previously argued that inadequate information about the location of cases constitutes in itself an unanswered research question on yaws. The most recent figures reported to WHO sum up about 100,000 new cases ${ }^{8}$ of yaws yearly, but cases reported are incomplete and very limited, so we are nowhere near the full extent of the problem. The resurgence of the disease and recent developments using a single dose of oral azithromycin have renewed optimism that eradication can be achieved through a comprehensive large-scale treatment strategy. To eradicate yaws, there is a need to intervene everywhere that the disease occurs, no matter how remotely located or difficult to access. Further mapping of populations in which yaws is known or suspected to be endemic should be considered a priority in the yaws eradication agenda.

\section{Hard-to-reach populations and migration}

Difficult-to-reach populations add to the complexities already facing the international community in ensuring that those affected are effectively reached. Residual transmission in some yaws-eliminating countries might be concentrated in a few hard-to-reach populations. Delivery of services to these hot spots can be challenging because their identities vary by setting and their members often face substantial barriers to health care access. Hard-to-reach populations, including ethnic or political minority groups, are typically impoverished and mobile, often driven to more remote areas by marginalization, safety concerns, and economic opportunities.

One of the hot spots for yaws in Africa is the humid forested region in the borders between Cameroon, Central African Republic, Democratic Republic of the Congo, and Republic of the Congo. This area is inhabited by indigenous populations who live in poor areas with little or no access to health care. Recent serological (Boua B, personal communication, March, 2013) surveys have reported serological prevalence rates between $20 \%$ and $86 \%$ in these communities. There are several challenges in bringing health care to the indigenous populations: 1) difficulty of access to the communities; 2) high cost of medical missions to their communities; 3) lack of health services to integrate activities; and 4) unhygienic and crowded living conditions in deep humid forest, which is favorable for yaws. Equitable access to yaws prevention and treatment should be addressed early in the eradication effort.
Migration and imported yaws is the other major threat to achievement and maintenance of elimination, with greatest risk for countries neighboring high-endemic areas. With the ever-increasing movement of people, more instances of yaws reintroduction to yaws-eliminating areas need to be anticipated for development of appropriate surveillance and response systems.

\section{Serological cross-reactivity of syphilis and yaws}

The infection with one T. pallidum subspecies does not protect against infection with another. Thus, it is quite possible that syphilis and yaws could exist in the same community. Several studies have reported the presence of syphilis in some countries where yaws is endemic (eg, Papua New Guinea), ${ }^{46,47}$ and, in a number of countries of West Africa, high prevalence rates for syphilis have been found in pregnant women based on rapid treponemal tests. ${ }^{48}$ In the past syphilis was generally considered to be more prevalent in large towns, where yaws is usually absent. The wearing of more clothing by children, and perhaps less crowded conditions at night might be contributory factors. Moreover, the two diseases affect different age-groups. Yaws is usually contracted in childhood and infectious lesions are infrequent after the age of 30 years.

Nevertheless, the possibility that in some areas yaws and syphilis are both being actively transmitted serves as a note of caution. Current serological tests are unable to discriminate among the agents of the human treponematoses. The coexistence of venereal and nonvenereal treponemal infections might affect the accuracy of epidemiological surveillance.

\section{Conclusion}

Yaws, a disease once again targeted for eradication after the 1950s-1960s WHO-United Nations Children's Fund sponsored campaign, is now being reported in at least 12 countries in tropical areas of Africa, Southeast Asia, and the Pacific. Yet its epidemiology is not yet fully understood. The extent of the yaws burden remains unclear due to various factors, including low completeness/timeliness of reporting, the inconsistent reporting of cases across countries because of the status of non-notifiable disease, misdiagnosis, and lack of accuracy of data. Moreover, the fact that the disease affects mostly remote communities, migrant groups, and minority groups with difficult or no access to health care (with the ensuing underreporting) as well as the cross-seroreactivity between yaws and syphilis further impacts the validity of population estimates of the disease prevalence among others. More effort is needed in order to refine accuracy of data currently being 
reported. A better characterization of the epidemiology of yaws globally is likely to positively impact the planning and implementation of yaws eradication.

\section{Acknowledgments}

We are grateful to Dr Bernard Boua and the Central African Republic National program for NTDs, to Dr Gilbert Ayelo and the National program for Buruli Ulcer of Benin, to Ms Wendy Houinei and the Papua New Guinea National Department of Health, to Dr Fasihah Taleo and the Neglected Tropical Diseases Program, Public Health Directorate, Vanuatu Health Department, and to Dr Christina Widaningrum and the Ministry of Health of Indonesia for granting us permission to use their unpublished data on cases of yaws.

\section{Disclosure}

The authors report no conflicts of interest in this work.

\section{References}

1. Mitjà $\mathrm{O}$, Asiedu K, Mabey D. Yaws. Lancet. 2013;381:763-773.

2. World Health Organization. Summary Report of the Consultative Meeting on Eradication of Yaws, March 5-7, 2012, Morges, Switzerland. Geneva: World Health Organization; 2012. Available from: http://apps. who.int/ iris/bitstream/10665/75528/1/WHO_HTM_NTD_IDM_2012.2_eng. pdf. Accessed February 5, 2013.

3. Mitjà $\mathrm{O}$, Hays R, Ipai $\mathrm{A}$, et al. Single-dose azithromycin versus benzathine benzylpenicillin for treatment of yaws in children in Papua New Guinea: an open-label, non-inferiority, randomised trial. Lancet. 2011;379:342-347.

4. Hill KR. Non-specific factors in the epidemiology of yaws. Bull World Health Organ. 1953;8:17-51.

5. Saunders G, Chambers H, Rerrie J. Annual report of the Jamaica Yaws Commission for 1936. Kingston, Jamaica: 1936.

6. Perine PL, Hopkins DR, Niemel PLA, St John RK, Causse G, Antal GM. Handbook of endemic treponematoses: Yaws, Endemic Syphilis, and Pinta. Geneva: World Health Organization; 1984.

7. Selected references on yaws [webpage on the Internet]. Geneva: World Health Organization. Available from: http://www.who.int/yaws/resources/ historic_documents/en/index.html. Accessed February 5, 2013.

8. Global Health Observatory Data Repository: Yaws [webpage on the Internet]. Geneva: World Health Organization; 2013. Available from: http://apps.who.int/gho/data/node.main.NTDYAWS?lang=en. Accessed February 5, 2013.

9. Hackett CJ. Extent and nature of the yaws problem in Africa. Bull World Health Organ. 1953;8:129-182.

10. World Health Organization. Resolutions and decisions: WHA2.36 Bejel and Other Treponematoses. Geneva: World Health Organization; 1949. Available from: http://www.who.int/neglected_diseases/mediacentre/ WHA_2.36_Eng.pdf. Accessed February 5, 2013.

11. World Health Organization. International Work on Endemic Treponematoses and Venereal Infections. Geneva: World Health Organization; 1965.

12. Anselmi M, Moreira JM, Caicedo C, Guderian R, Tognoni G. Community participation eliminates yaws in Ecuador. Trop Med Int Health. 2003;8:634-638.

13. No authors listed. Elimination of yaws in India. Wkly Epidemiol Rec. 2008;83(15):125-132. English, French.

14. Coldiron M, Obvala D, Mouniaman-Nara I, Pena J, Blondel C, Porten K. [The prevalence of yaws among the Aka in the Congo]. Med Sante Trop. 2013;23(2):231-232. French.
15. Manirakiza A, Boas SV, Beyam N, et al. Clinical outcome of skin yaws lesions after treatment with benzathine benzylpenicillin in a pygmy population in Lobaye, Central African Republic. BMC Res Notes. 2011;4:543.

16. Widy-Wirski R, D’Costa J, Meheus A. [Prevalence of yaws in pygmies of the Central African Republic]. Ann Soc Belg Med Trop. 1980;60(1): 61-67. French.

17. Cirera P, Palisson MJ, Pinerd G, Jaeger G. [Treponemal serology in a Bi-Aka pygmy population of Central Africa]. Bull Soc Pathol Exot Filiales. 1977;70(1):32-36. French.

18. Hervé V, Kassa Kelembho E, Normand P, Georges A, Mathiot C, Martin P. [Resurgence of yaws in Central African Republic. Role of the Pygmy population as a reservoir of the virus]. Bull Soc Pathol Exot. 1992;85:342-346. French.

19. Louis FJ, Miailhes P, Trébucq A, Maubert B, Louis JP. Le pian chez les Pygmées, indicateur d'une régression de l'accès aux soins en Afrique Centrale. Cahier Santé. 1993;3:128-132. French.

20. Touré B, Koffi NM, Assi KP, Ake O, Konan DJ. [Yaws in Cote d'lvoire: health problem forgotten and neglected]. Bull Soc Pathol Exot. 2007;100:130-132. French.

21. Edorh AA, Siamevi EK, Adanlete FA, et al. [Resurgence of endemic yaws in Togo. Cause and eradication approach]. Bull Soc Pathol Exot. 1994;87:17-18. French.

22. Gerstl S, Kiwila G, Dhorda M, et al. Prevalence study of yaws in the Democratic Republic of Congo using the lot quality assurance sampling method. PLoS One. 2009;4(7):e6338.

23. Akogun OB. Yaws and syphilis in the Garkida area of Nigeria. Zentralbl Bakteriol. 1999;289:101-107.

24. Nnoruka EN. Skin diseases in south-east Nigeria: a current perspective. Int J Dermatol. 2005;44:29-33.

25. dos Santos MM, Amaral S, Harmen SP, Joseph HM, Fernandes JL, Counahan ML. The prevalence of common skin infections in four districts in Timor-Leste: a cross sectional survey. BMC Infect Dis. 2010;10:61.

26. Engelkens HJ, Stolz E. A small yaws survey on the island of Sumatra, Indonesia. Acta Leiden. 1992;60(2):19-29.

27. Noordhoek GT, Engelkens HJ, Judanarso J, et al. Yaws in West Sumatra, Indonesia: clinical manifestations, serological findings and characterisation of new Treponema isolates by DNA probes. Eur J Clin Microbiol Infect Dis. 1991;10(1):12-19.

28. Backhouse JL, Hudson BJ, Hamilton PA, Nesteroff SI. Failure of penicillin treatment of yaws on Karkar Island, Papua New Guinea. Am J Trop Med Hyg. 1998;59(3):388-392.

29. Fegan D, Glennon M, Macbride-Stewart G, Moore T. Yaws in the Solomon Islands. J Trop Med Hyg. 1990;93:52-57.

30. Manning LA, Ogle GD. Yaws in the periurban settlements of Port Moresby, Papua New Guinea. P N G Med J. 2002;45:206-212.

31. Mitjà $\mathrm{O}$, Hays $\mathrm{R}$, Ipai $\mathrm{A}$, et al. Outcome predictors in treatment of yaws. Emerg Infect Dis. 2011;17:1083-1085.

32. Alemaena O. Yaws situation in the Solomon Islands. Southeast Asian J Trop Med Public Health. 1986;17:14-18.

33. Ministry of Health, Division of Planning and Policy, National Health Statistics Office-Solomon Islands. Annual health report 2007. Technical Report Solomon Islands 2008.

34. Fegan D, Glennon MJ, Thami Y, Pakoa G. Resurgence of yaws in Tanna, Vanuatu: time for a new approach? Trop Doct. 2010;40:68-69.

35. Harris M, Nako D, Hopkins T, et al. Yaws infection in Tanna, Vanuatu 1989. Southeast Asian J Trop Med Public Health. 1991;22: 113-119.

36. de Noray G, Capuano C, Abel M. [Campaign to eradicate yaws on Santo island, Vanuatu 2001]. Med Trop (Mars). 2003;63:159-162. French.

37. Guerrier G, Marcon S, Garnotel L, et al. Yaws in Polynesia's Wallis and Futuna Islands: a seroprevalence survey. $N$ Z Med J. 2011; 124(1333):29-31.

38. Scolnik D, Aronson L, Lovinsky R, et al. Efficacy of a targeted, oral penicillin-based yaws control program among children living in rural South America. Clin Infect Dis. 2003;36:1232-1238.

39. Guderian RH, Guzman JR, Calvopiña M, Cooper P. Studies on a focus of yaws in the Santiago Basin, province of Esmeraldas, Ecuador. Trop Geogr Med. 1991;43(1-2):142-147. 
40. Anselmi M, Araujo E, Narváez A, Cooper PJ, Guderian RH. Yaws in Ecuador: impact of control measures on the disease in the Province of Esmeraldas. Genitourin Med. 1995;71:343-346.

41. World Health Organization Regional Office for South Eats Asia. Yaws eradication in the South East Asia region. Report of an intercountry workshop. Bali, Indonesia, 19-21 July, 2006. Tech Rep WHO. New Delhi, India, 2006.

42. Geizer I. Yaws in the Western Pacific region: an overview. Southeast Asian J Trop Med Public Health. 1986;17:8-13.

43. Guerrier G, Marcon S, Garnotel L. Yaws in Polynesis's Wallis and Futuna Islands: a seroprevalence survey. NZ Med J. 2011;124:29-31.

44. Garner MF, Backhouse JL, Moddie PM, Tibbs GJ. Treponemal infection in the Australian Northern Territory aborigines. Bull WHO. $1972 ; 46: 285-293$
45. Hopkins DR. Yaws in the Americas, 1950-1975. J Infect Dis. 1977;136: $548-554$.

46. Hudson BJ, van der Meijden WI, Lupiwa T, et al. A survey of sexually transmitted diseases in five STD clinics in Papua New Guinea. $P N G$ Med J. 1984;37:152-160.

47. Bruce E, Bauai L, Masta A, et al. A cross-sectional study of reported symptoms for sexually transmissible infections among female sex workers in Papua New Guinea. Sex Health. 2010;7:71-76.

48. Greenwood AM, D'Allessandro U, Sisay F, Greenwood BM. Treponemal infection and the outcome of pregnancy in a rural area of the Gambia, West Africa. J Infect Dis. 1992;166:842-846.
Clinical Epidemiology

\section{Publish your work in this journal}

Clinical Epidemiology is an international, peer-reviewed, open access journal focusing on disease and drug epidemiology, identification of risk factors and screening procedures to develop optimal preventative initiatives and programs. Specific topics include: diagnosis, prognosis, treatment, screening, prevention, risk factor modification, systematic

Submit your manuscript here: http://www.dovepress.com/clinical-epidemiology-journal

\section{Dovepress}

reviews, risk \& safety of medical interventions, epidemiology \& biostatical methods, evaluation of guidelines, translational medicine, health policies \& economic evaluations. The manuscript management system is completely online and includes a very quick and fair peer-review system, which is all easy to use. 